OPEN ACCESS

Edited by:

Elias Adel Rahal,

American University of Beirut, Lebanon

Reviewed by:

Tongqiang Zhang,

Tianjin Children's Hospital, China James Daubenspeck, University of Alabama at Birmingham, United States

*Correspondence: Yuanyuan Zhang chzyy@zju.edu.cn Zhimin Chen zmchen@zju.edu.cn

Specialty section: This article was submitted to Microbes and Innate Immunity, a section of the journal

Frontiers in Cellular and Infection Microbiology

Received: 29 March 2021 Accepted: 28 June 2021 Published: 15 July 2021

Citation:

Zheng Y, Hua L, Zhao Q, Li M, Huang $M$, Zhou $Y$, Wang $Y$, Chen $Z$ and Zhang $Y$ (2021) The Level of D-Dimer Is Positively Correlated With the Severity of Mycoplasma pneumoniae Pneumonia in Children. Front. Cell. Infect. Microbiol. 11:687391.

doi: 10.3389/fcimb.2021.687391

\section{The Level of D-Dimer Is Positively Correlated With the Severity of Mycoplasma pneumoniae Pneumonia in Children}

\author{
Yan Zheng ${ }^{1,2}$, Lingling Hua ${ }^{1,3}$, Qiannan Zhao ${ }^{1}$, Mengyao Li $^{1}$, Meixia Huang ${ }^{1}$, \\ Yunlian Zhou ${ }^{1}$, Yingshuo Wang ${ }^{1}$, Zhimin Chen ${ }^{1 *}$ and Yuanyuan Zhang ${ }^{1 *}$ \\ ${ }^{1}$ Department of Pulmonology, Children's Hospital Zhejiang University School of Medicine, National Clinical Research Center \\ for Child Health, Hangzhou, China, 2 Department of Pediatrics, The Quzhou Affiliated Hospital of Wenzhou Medical University, \\ Quzhou People's Hospital, Quzhou, China, ${ }^{3}$ Department of Pediatrics, Ningbo Women and Children's Hospital, Ningbo, China
}

Objective: Mycoplasma pneumoniae pneumonia (MPP) is an important disease in children. Studies have demonstrated that the levels of D-dimer are elevated in some children with MPP, especially those with thrombotic complications. However, the potential association between MPP and D-dimer remains unclear. In our study, we sought to explore the relationship between the levels of plasma D-dimer and clinical characteristics of MPP patients.

Methods: Retrospective analysis was conducted on 356 patients who were hospitalized in our hospital for MPP between January 1, 2017, and December 31, 2019. According to the peak value of D-dimer, patients were divided into three groups: the normal group (D-dimer $<0.55 \mathrm{mg} / \mathrm{L}$ ), the mild-moderately elevated group (D-dimer $0.55-5.5 \mathrm{mg} / \mathrm{L}$ ) and the severely elevated group (D-dimer $>5.5 \mathrm{mg} / \mathrm{L}$ ). The demographic and clinical information, radiological findings, laboratory data, and treatments of patients were compared among different groups.

Results: 106 patients were in the normal group, 204 patients were in the mild-moderately elevated group, and 46 patients were in the severely elevated group. More severe clinical and radiographic manifestations, longer length of fever, hospital stay and antibiotic therapy duration, higher incidences of extra-pulmonary complications, refractory MPP (RMPP), severe MPP (SMPP) were found in the elevated group, when compared with the normal group $(P<0.01)$. Meanwhile, we found that the percentage of neutrophil $(\mathrm{N} \%)$ and $\mathrm{CD}_{8}{ }^{+}$ lymphocyte $\left(\mathrm{CD}_{8}{ }^{+} \%\right)$, C-reactive protein $(\mathrm{CRP})$, lactate dehydrogenase $(\mathrm{LDH})$, interleukin (IL)-6, IL-10, and interferon-gamma (IFN- $\gamma$ ) trended higher with increasing D-dimer, whereas the percentage of lymphocyte $(\mathrm{L} \%)$ and prealbumin $(\mathrm{PAB})$ trended lower $(P<0.01)$. In addition, the proportions of patients requiring oxygen therapy, glucocorticoid, bronchoscopy, immunoglobulin use, thoracentesis, or ICU admission were significantly higher in the severely elevated group than those in the other two groups $(P<0.01)$. Correlation analysis showed that N\%, L\%, CRP, LDH, IL-10, length of 
fever, length of stay, and length of antibiotic therapy had strong correlations with the level of D-dimer.

Conclusions: MPP patients with higher levels of D-dimer had more severe clinical manifestations and needed longer duration of treatment, which might be closely related to the severity of lung inflammation after MP infection.

Keywords: Mycoplasma pneumoniae, pneumonia, D-dimer, children, severity

\section{INTRODUCTION}

Mycoplasma pneumoniae (MP) is one of the most important microorganisms that caused community-acquired pneumonia (CAP) in children (Jain et al., 2015; Meyer Sauteur et al., 2020). It may be responsible for about $4 \%$ to $8 \%$ of CAP during periods of endemicity, whereas it could cause up to $20 \%$ to $40 \%$ of CAP in the general population during epidemics (Waites et al., 2017). In general, Mycoplasma pneumoniae pneumonia (MPP) is recognized as a self-limited disease, but recently, some researchers reported that it could cause pulmonary and extrapulmonary complications, or even life-threatening situations, such as necrotizing pneumonitis, myocarditis, hemolytic anemia, acute pancreatitis, and so on (Poddighe, 2018). Furthermore, several recent studies show that MPP patients could have concurrent pulmonary embolism, cerebral infarction, spleen infarction, and other systemic thrombotic diseases (Park et al., 2012; Kang et al., 2016; Mélé and Turc, 2018; Chen et al., 2020). The pathogenic mechanisms of MP infection may be related to the cytoadherence, intracellular localization, cytotoxicity, inflammation, and so on, which is still elusive (Waites and Talkington, 2004).

D-dimer is a soluble fibrin degradation product that results from the ordered breakdown of thrombi by the fibrinolytic system (Weitz et al., 2017). Numerous studies have reported that elevated D-dimer is associated with thrombotic diseases (Goldenberg et al., 2004; Nowak-Göttl et al., 2004). Consequently, D-dimer has been extensively used for the diagnosis of venous thromboembolism (VTE) (Adam et al., 2009). Recently, it has also been reported that the levels of D-dimer are related to the severity of coronavirus disease 2019 (COVID-19) (Sakka et al., 2020). However, few people had concentrated on the relationship between clinical manifestations of MPP and D-dimer.

In this study, we retrospectively analyzed the clinical characteristics of MPP patients with different degrees of Ddimer who were hospitalized in our hospital between January 1, 2017, and December 31, 2019, and explore the relationship between the levels of $\mathrm{D}$-dimer and clinical characteristics of MPP patients.

\section{METHODS}

\section{Study Population}

In this study, we retrospectively collected the clinical data of patients with MMP who were admitted to Children's hospital,
Zhejiang University School of Medicine between January 1, 2017, and December 31, 2019. The criteria for enrolling patients were as follows: (1) signs and symptoms indicative of CAP, including fever, cough, abnormal lung auscultation and new infiltrate(s) on chest radiograph; (2) had both positive results for MP RNA polymerase chain reaction (PCR) tests and positive results for MP-IgM (Witmer et al., 2007; Waites et al., 2017). Exclusion criteria were the follows (Zhang et al., 2014; Jin et al., 2018): (1) had positive results for other pathogens; (2) had received corticosteroids, intravenous immunoglobulin (IVIG), and anticoagulation therapy before admission; (3) with underlying diseases, such as chronic cardiac and pulmonary disease, rheumatic diseases, and immunodeficiency; (4) had incomplete medical records.

\section{Data Collection}

Demographic and clinical information, radiological findings, laboratory data, such as blood routine test, C-reactive protein (CRP), lactate dehydrogenase $(\mathrm{LDH}), \mathrm{D}$-dimer, prealbumin (PAB), subpopulations of $\mathrm{T}$ lymphocytes, immunoglobulins, cytokines were retrospectively collected from all patients by reviewing their electronic medical records. During the hospitalization, clinical signs and symptoms of patients were obtained, including body temperature, respiratory rates, extrapulmonary complications (Poddighe, 2018,) (Witmer et al., 2007; Zhou et al., 2014), and so on. All patients underwent chest radiography during the illness, confirming unequivocal focal or segmental infiltration with or without pleural effusion. Although patients had progressive symptoms, suspected complications, clinical deterioration, or persistent fever after appropriate antibiotic therapy, chest CT scans were performed. The large lesion was defined as the extent of infiltration on chest imaging more than one third of the lung (Uehara et al., 2011).

Severe MPP (SMPP) was defined as MPP with any one of the follows: (1) a poor general condition; (2) fastidium or dehydration; (3) disturbance of consciousness; (4) an increased respiratory rate (infants $>70$ breaths/min and older children $>50$ breaths/min); (5) dyspnea; (6) cyanosis; (7) extent of infiltration on chest $\mathrm{X}$-ray $\geq 2 / 3$ of one lung or multilobe involvement; (8) extra-pulmonary complications; (9) pleural effusion; (10) oxygen saturation in room air $\leq 92 \%$ (Subspecialty Group of Respiratory Diseases, 2013). Refractory MPP (RMPP) was diagnosed based on the presence of persistent fever and clinical, as well as radiological deterioration after azithromycin treatment for 7 days or longer (Tamura et al., 2008; Subspecialty Group of Respiratory Diseases, 2013). The indications for oxygen 
therapy, bronchoscopy, glucocorticoid, and mechanical ventilation were evaluated according to the guidelines for management of community-acquired pneumonia in children in China (Subspecialty Group of Respiratory Diseases, 2013).

\section{Sample Detection}

Nasopharyngeal aspirate/swab specimens were routinely tested within 24 hours after admission. Peripheral blood samples were obtained on admission for detecting the laboratory data, and the abnormal values of D-dimer and inflammatory indicators, such as WBC, CRP, cytokines, and so on, were detected every 3 to 5 days thereafter.

Serum cytokines were routinely measured because this would be, to some extent, helpful for therapy decisions, and the costs were not expensive. We detected the concentrations of interleukin (IL)-2, IL-4, IL-6, IL-10, tumor necrosis factor-alpha (TNF- $\alpha$ ), and interferon-gamma (IFN- $\gamma$ ) in serum by a CBA HumanTh1/Th2 Cytokine Kit II (BD Biosciences, San Diego, CA, USA). The value of D-dimer was determined via INNOVANCE D-dimer (SIEMENS, Marburg, Germany), the normal value was less than $0.55 \mathrm{mg} / \mathrm{L}$. The MP IgM was determined via a specific Anti-MP ELISA assay (EUROIMMUN, Luebeck, Germany), the absorbance above 1.1 was determined positive. MP RNA detection was performed on an ABI 7500 detection system via SAT-MP Assay Kit (Rendu Biotechnology Co., Ltd, Shanghai, China). All steps were performed according to the manufacturer's instructions and previous studies (Zhang et al., 2016; Li et al., 2017).

\section{Ethics}

The study was approved by the ethics committee of the Children's Hospital, Zhejiang University School of Medicine (2019-RIB-058). And the data from patients were collected anonymously.

\section{Statistical Analysis}

SPSS 20.0 (IBM Corp., Armonk, NY, USA) was used for statistical analysis. Continuous data are shown as median (25th to 75 th percentile). Categorical data were shown as number (\%). The Kruskal-Wallis- $\mathrm{H}(\mathrm{K}-\mathrm{W}-\mathrm{H})$ method was used to compare differences in continuous variables among multiple groups. The Mann-Whitney U-test was used to compare differences in continuous variables between two groups. Pearson's chi-square test was used to analyze differences between categorical variables. Spearman rank-correlation coefficients were used to describe the association between different variables and D-dimer. Statistical significance was defined as $\mathrm{P}<0.05$.

\section{RESULTS}

\section{General Information of Patients}

From January 1, 2017, to December 31, 2019, a total of 356 patients admitted to our hospital for MPP were enrolled in the study. All patients had positive MP PCR tests and serological detection. According to the peak value of D-dimer (Huang et al., 2021), they were divided into three groups, the normal group (D-dimer <0.55 mg/L, $\mathrm{n}=106$ ), the mild-moderately elevated group (D-dimer $0.55-5.5 \mathrm{mg} / \mathrm{L}, \mathrm{n}=204$ ) and the severely elevated group (more than 10 times higher than the normal range, D-dimer $>5.5 \mathrm{mg} / \mathrm{L}, \mathrm{n}=46$ ). As shown in Table 1, the median age in the normal group was 4.1 years (range, 2.1-6.1), younger than that in the mild-moderately elevated group 5.8 years (range, 4.3-7.0) and the severely elevated group 6.1 years (range, 4.6-8.0) $(P<0.01)$, but no difference was found in gender distribution $(P>0.05)$.

\section{Clinical Characteristics of Patients}

The most common symptoms of MPP were cough (100.0\%) and fever $(98.0 \%)$. Chest pain (1.7\%) was rare, and 62 patients (17.4\%) presented with wheezing. As shown in Table 1, the higher incidence of fever and the lower incidence of wheezing were found in the mild-moderately or severely elevated group than those in the normal group $(P<0.01)$. We also found that the total length of fever, the total length of antibiotic therapy, the length of stay, and the incidences of RMPP and SMPP were significantly different among the three groups $(P<0.01)$. Interestingly, there were 14 patients (13.2\%) with extrapulmonary complications in the normal group, 72 patients (35.3\%) in the mild-moderately elevated group, and 33 patients $(71.7 \%)$ in the severely elevated group, which showed significant differences among these three groups $(P<0.01)$. Moreover, the total length of fever, the total length of antibiotic therapy, the length of stay, and the incidence of extra-pulmonary complications, RMPP, SMPP were increased with the level of D-dimer $(P<0.01)$.

\section{Laboratory Findings of Patients}

Laboratory findings of patients on admission were shown in Table 2. Besides D-dimer, the WBC, percentage of peripheral neutrophils (N\%) and lymphocytes (L\%), platelet (PLT), CRP, $\mathrm{LDH}, \mathrm{PAB}$, percentage of $\mathrm{CD}_{4}{ }^{+}$and $\mathrm{CD}_{8}{ }^{+} \mathrm{T}$ lymphocytes $\left(\mathrm{CD}_{4}{ }^{+} \%, \mathrm{CD}_{8}{ }^{+} \%\right)$, IgA, IL-6, IL-10, TNF- $\alpha$, IFN- $\gamma$ also differed significantly among the three groups $(P<0.01)$, but there were no statistically significant differences in hemoglobin, percentage of $\mathrm{CD}_{3}{ }^{+} \mathrm{T}$ lymphocytes $\left(\mathrm{CD}_{3}{ }^{+} \%\right)$, IL-2, IL-4, IgG, IgM, and IgE $(P>0.05)$. Furthermore, we found that N\%, CRP, $\mathrm{LDH}, \mathrm{CD}_{8}{ }^{+} \%$, IL-6, IL-10, and IFN- $\gamma$ were significantly increased in the elevated group, especially in the severely elevated group than those in the normal group, which were in line with the levels of D-dimer; whereas the $\mathrm{L} \%$ and PAB decreased with the levels of D-dimer.

\section{Radiographic Features of Patients}

As shown in Table 3, we found that there were significant differences in radiographic features among the three groups $(P<0.01)$. With the increase of $\mathrm{D}$-dimer, the incidences of pleural effusion $(26.4 \%$ vs. $64.2 \%$ vs. $93.5 \%, P<0.01)$, lobar atelectasis $(3.8 \%$ vs. $33.3 \%$ vs. $63.0 \%, P<0.01)$, pulmonary consolidation $(22.6 \%$ vs. $57.8 \%$ vs. $87.0 \%, P<0.01)$, large lesions ( $18.9 \%$ vs. $33.5 \%$ vs. $55.6 \%, P<0.01$ ) were all significantly increased. As for necrotizing pneumonia, we found that it was likely to occur in the severely elevated group $(23.9 \%, P<0.01)$, and there was no difference between the normal group and the 
TABLE 1 | Demographic and clinical characteristics of patients with MPP.

\begin{tabular}{|c|c|c|c|c|}
\hline Clinical information & Normal group $(n=106)$ & Mild-moderately elevated group $(n=204)$ & Severely elevated group $(n=46)$ & $P$-value \\
\hline Sex (male/female) & $56 / 50$ & $91 / 113$ & $21 / 25$ & 0.397 \\
\hline Age, years & $4.1(2.1 \sim 6.1)$ & $5.8(4.3 \sim 7.0)^{\text {a\# }}$ & $6.1(4.6 \sim 8.0){ }^{b \#}$ & 0.000 \\
\hline \multicolumn{5}{|l|}{ Clinical presentation, n (\%) } \\
\hline Fever & $100(94.3 \%)$ & $203(99.5 \%)$ a\# & $46(100.0 \%){ }^{b \#}$ & 0.010 \\
\hline Cough & $106(100.0 \%)$ & $204(100.0 \%)$ & $46(100.0 \%)$ & 1.000 \\
\hline Wheezing & $34(32.1 \%)$ & $25(12.3 \%)^{\text {a\# }}$ & $3(6.5 \%)$ & 0.000 \\
\hline Chest pain & $2(1.9 \%)$ & $3(1.5 \%)$ & $1(2.2 \%)$ & 1.000 \\
\hline Extra-pulmonary complications, n (\%) & $14(13.2 \%)$ & $72(35.3 \%)^{\text {a\# }}$ & $33(71.7 \%)$ b\#,c\# & 0.000 \\
\hline Digestive system & $7(6.6 \%)$ & $45(22.1 \%)$ & $12(26.1 \%)$ & \\
\hline Cardiovascular system & $2(1.9 \%)$ & $9(4.4 \%)$ & 5 (10.9\%) & \\
\hline Neurologic system & $2(1.9 \%)$ & $4(2.0 \%)$ & $2(4.3 \%)$ & \\
\hline Hematologic system & $1(0.9 \%)$ & $2(1.0 \%)$ & $3(6.5 \%)$ & \\
\hline Skin and Mucosae & $1(0.9 \%)$ & $3(1.5 \%)$ & $0(0.0 \%)$ & \\
\hline Multi-systems & $1(0.9 \%)$ & $9(4.4 \%)$ & $11(23.9 \%)$ & \\
\hline Length of fever, days & $7.3 \pm 3.9$ & $10.2 \pm 3.1$ a\# & 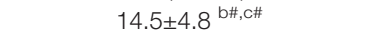 & 0.000 \\
\hline Length of stay, days & $6.0(4.0 \sim 7.0)$ & $7.0(5.0 \sim 10.0)^{a \#}$ & $13.0(10.0 \sim 18.0)^{\mathrm{b \# ,c \#}}$ & 0.000 \\
\hline Length of antibiotic therapy days & $10.5(8.0 \sim 13.3)$ & $12.0(10.0 \sim 16.0)^{a \#}$ & $19.0(15.0 \sim 25.0)^{b \#, c \#}$ & 0.000 \\
\hline RMPP, n (\%) & $7(6.6 \%)$ & $85(41.7 \%)^{\text {a\# }}$ & $40(87.0 \%)$ & 0.000 \\
\hline SMPP, n (\%) & 68 (64.2\%) & $176(86.3 \%)$ a\# & $46(100.0 \%){ }^{b \#, c \#}$ & 0.000 \\
\hline
\end{tabular}

Data are presented as median $\left(25^{\text {th }} \sim 75^{\text {th }}\right.$ percentile), or number (percentage). ${ }^{\#} P<0.01$; ${ }^{a}$ Compared between normal group and mild-moderately elevated group; ${ }^{b}$ compared between normal group and severely elevated group; ${ }^{\circ}$ compared between mild-moderately elevated group and severely elevated group.

Digestive system complications: hepatic impairment, hepatomegaly.

Cardiovascular system complications: myocardial damage, pericardial effusion, Kawasaki disease.

Neurologic system complications: encephalitis, Guillain-Barre's syndrome.

Hematologic system complications: anemia, cytopenia.

Skin and Mucosae complications: unspecific rashes, Stevens-Johnson syndrome.

RMPP, Refractory mycoplasma pneumonia pneumonia; SMPP, Severe mycoplasma pneumonia pneumonia.

TABLE 2 | Laboratory findings of patients with MPP on admission.

\begin{tabular}{|c|c|c|c|c|}
\hline Laboratory information & Normal group $(n=106)$ & Mild-moderately elevated group $(n=204)$ & Severely elevated group $(n=46)$ & $P$-value \\
\hline \multicolumn{5}{|l|}{ Routine blood test } \\
\hline White blood cell $\left(\times 10^{9} / \mathrm{L}\right)$ & $7.72(6.28 \sim 9.79)$ & $7.33(5.79 \sim 9.36)$ & $8.22(6.72 \sim 10.65)^{\mathrm{c} *}$ & 0.048 \\
\hline Neutrophil, \% & $57.7(43.5 \sim 65.2)$ & $65.4(55.9 \sim 71.8)^{a \#}$ & 75.9 (66.8 81.2) & 0.000 \\
\hline Lymphocyte, \% & $31.2(25.9 \sim 44.8)$ & $24.7(18.9 \sim 32.5)^{\text {a\# }}$ & $17.2(12.6 \sim 25.7) \mathrm{b \# ,c \#}$ & 0.000 \\
\hline Hemoglobin, g/L & $122(113 \sim 128)$ & 122 (116 129) & 119 (114 129) & 0.197 \\
\hline Platelet $\left(\times 10^{9} / \mathrm{L}\right)$ & 336 (267 405) & $288(222 \sim 361)^{\text {a\# }}$ & $262(197 \sim 336){ }^{b \#}$ & 0.000 \\
\hline $\mathrm{CRP}, \mathrm{mg} / \mathrm{L}$ & $3.4(0.5 \sim 12.7)$ & $15.1(6.2 \sim 37.0)^{\text {a\# }}$ & $56.2(23.4 \sim 95.3)$ b\#,c\# & 0.000 \\
\hline $\mathrm{LDH}, \mathrm{IU} / \mathrm{L}$ & 334 (272 438) & $440(348 \sim 591)$ a\# & 678(542 944) b\#,c\# & 0.000 \\
\hline$P A B, g / L$ & $0.11(0.10 \sim 0.14)$ & $0.10(0.08 \sim 0.13)^{a \#}$ & $0.08(0.06 \sim 0.12){ }^{b \#, c \#}$ & 0.000 \\
\hline \multicolumn{5}{|c|}{ Subpopulations of T lymphocytes, \% } \\
\hline $\mathrm{CD}_{3}$ & 65.55 (58.48 74.84) & 66.68 (59.92 74.23) & 63.55 (54.74 72.39) & 0.156 \\
\hline $\mathrm{CD}_{4}$ & 36.98 (31.64 43.66) & 36.15 (29.90 42.09) & $28.23(23.76 \sim 36.60))^{b \#, c \#}$ & 0.000 \\
\hline $\mathrm{CD}_{8}$ & $20.92(16.70 \sim 26.28)$ & 23.33 (19.44 28.99) a\# & $26.48(22.95 \sim 33.09)^{b \#, c \#}$ & 0.000 \\
\hline \multicolumn{5}{|l|}{ Total Immunoglobulin (lg) } \\
\hline $\operatorname{lgG}, \mathrm{g} / \mathrm{L}$ & $8.80(7.10 \sim 10.73)$ & 8.95 (7.60 10.70) & 8.35 (7.18 10.63) & 0.475 \\
\hline $\lg A, g / L$ & $1.02(0.69 \sim 1.37)$ & $1.28(0.87 \sim 1.78)^{\text {a\# }}$ & $1.25(0.99 \sim 1.64)^{b \#}$ & 0.000 \\
\hline $\operatorname{lgM}, \mathrm{g} / \mathrm{L}$ & 1.57 (1.16 2.03) & 1.65 (1.22 2.24) & 1.55 (0.99 2.40) & 0.229 \\
\hline $\operatorname{lgE}, \mathrm{IU} / \mathrm{ml}$ & 96.9 (37.6 218.5) & 93.9 (38.4 290.5) & 99.9 (51.1 287.3) & 0.855 \\
\hline \multicolumn{5}{|l|}{ Cytokines, pg/ml } \\
\hline IL-2 & $1.4(1.1 \sim 1.7)$ & $1.4(1.1 \sim 1.7)$ & $1.6(1.1 \sim 2.4)$ & 0.190 \\
\hline IL-4 & $2.0(1.7 \sim 2.3)$ & $2.0(1.6 \sim 2.5)$ & $2.2(1.4 \sim 2.8)$ & 0.593 \\
\hline IL-6 & $16.9(7.9 \sim 43.4)$ & $26.7(13.3 \sim 57.8)^{a *}$ & 59.5 (24.2 120.3) ${ }^{\mathrm{b \# ,c \#}}$ & 0.000 \\
\hline $\mathrm{IL}-10$ & $5.5(4.4 \sim 7.6)$ & $8.5(6.0 \sim 12.0)^{\text {a\# }}$ & $9.4(7.1 \sim 16.0)^{b \#, C *}$ & 0.000 \\
\hline TNF- $\alpha$ & $2.5(1.8 \sim 4.6)$ & $2.3(1.8 \sim 4.1)$ & $1.7(1.0 \sim 2.6)^{\mathrm{b \# ,c \#}}$ & 0.000 \\
\hline $\mathrm{IFN}-\gamma$ & $3.2(2.2 \sim 4.8)$ & $5.1(2.8 \sim 10.8)^{a \#}$ & $8.4(4.0 \sim 40.1)^{b \#, c \star}$ & 0.000 \\
\hline D-dimer, mg/L & $0.37(0.25 \sim 0.44)$ & $1.26(0.82 \sim 2.07)^{a \#}$ & $7.68(6.22 \sim 14.16){ }^{b \#, c \#}$ & 0.000 \\
\hline
\end{tabular}

Data are presented as the median $\left(25^{\text {th }}-75^{\text {th }}\right.$ percentile). ${ }^{*} P<0.05,{ }^{\#} P<0.01 ;{ }^{a}$ Compared between normal group and mild-moderately elevated group; ${ }^{b}$ Compared between normal group and severe elevated group; ${ }^{C}$ Compared between mild-moderately elevated group and severely elevated group. CRP, C-reactive protein; LDH, Lactate dehydrogenase; PAB, Prealbumin; IL-2, Interleukin 2; IL-4, Interleukin 4; IL-6, Interleukin 6; IL-10, Interleukin 10; TNF- $\alpha$, Tumor necrosis factor-alpha; IFN- $\gamma$, Interferon-gamma. 
TABLE 3 | Radiographic features of patients with MPP.

\begin{tabular}{|c|c|c|c|c|}
\hline Radiological features, n (\%) & Normal group $(n=106)$ & Mild-moderately elevated group $(n=204)$ & Severely elevated group $(n=46)$ & $P$-value \\
\hline Pleural effusion & $28(26.4 \%)$ & $131(64.2 \%)^{\text {a\# }}$ & $43(93.5 \%))^{b \#, c \#}$ & 0.000 \\
\hline Lobar atelectasis & $4(3.8 \%)$ & $68(33.3 \%)^{\text {a\# }}$ & $29(63.0 \%){ }^{b \#, c \#}$ & 0.000 \\
\hline Pulmonary consolidation & $24(22.6 \%)$ & $118(57.8 \%)^{\text {a\# }}$ & $40(87.0 \%){ }^{b \#, c \#}$ & 0.000 \\
\hline Large lesions & $20(18.9 \%)$ & $66(33.5 \%)^{\text {a\# }}$ & $30(55.6 \%){ }^{b \#, c \#}$ & 0.000 \\
\hline Necrotizing pneumonia & $1(0.9 \%)$ & 7 (3.4\%) & $11(23.9 \%)^{b \#, c \#}$ & 0.000 \\
\hline Embolism & $0(0.0 \%)$ & $0(0.0 \%)$ & $0(0.0 \%)$ & 1.000 \\
\hline
\end{tabular}

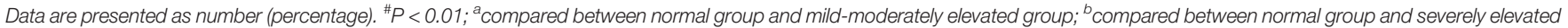
group; 'compared between mild-moderately elevated group and severely elevated group.

Large lesion was defined as the extent of infiltration on chest imaging more than 1/3 of the lung.

TABLE 4 | Treatments of patients with MPP.

\begin{tabular}{|c|c|c|c|c|}
\hline Treatments, $\mathrm{n}(\%)$ & Normal group $(n=106)$ & Mild-moderately elevated group $(n=204)$ & Severely elevated group $(n=46)$ & $P$-value \\
\hline Oxygen therapy & $28(26.4 \%)$ & $46(22.5 \%)$ & $24(52.2 \%)$ b\#,c\# & 0.000 \\
\hline Glucocorticoid & $51(48.1 \%)$ & $150(73.5 \%)^{\text {a\# }}$ & $44(95.7 \%)^{b \#, c \#}$ & 0.000 \\
\hline Bronchoscope & $21(19.8 \%)$ & $110(53.9 \%)$ a\# & $40(87.0 \%)$ b\#,c\# & 0.000 \\
\hline immunoglobulin & $3(2.8 \%)$ & $10(4.9 \%)$ & $14(30.4 \%)$ b\#,c\# & 0.000 \\
\hline $\mathrm{ICU}$ & $0(0.0 \%)$ & $2(1.0 \%)$ & $8(17.4 \%)$ b\#,c\# & 0.000 \\
\hline Mechanical ventilation & $0(0.0 \%)$ & $0(0.0 \%)$ & $0(0.0 \%)$ & 1.000 \\
\hline Thoracentesis & $0(0.0 \%)$ & $15(7.4 \%)^{\text {a\# }}$ & $19(41.3 \%)$ b\#,c\# & 0.000 \\
\hline
\end{tabular}

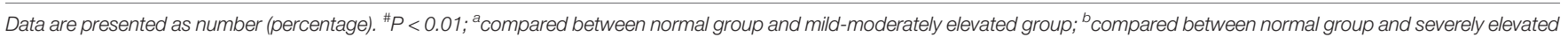
group; 'compared between mild-moderately elevated group and severely elevated group.

mild-moderately elevated group $(0.9 \%$ vs. $3.4 \%, P>0.05)$. However, none of the patients developed embolism in our study.

\section{Treatment of Patients}

In addition to antibiotics, patients received other treatments depending on the disease severity, such as oxygen therapy, glucocorticoid, bronchoscopy, immunoglobulin, thoracentesis, or ICU admission (as shown in Table 4). In our study, we found that patients in the severely elevated group received a higher proportion of oxygen therapy, glucocorticoid, bronchoscopy, immunoglobulin, thoracentesis, and ICU admission when compared with the other two groups $(P<0.01)$. Meanwhile, the usage rates of glucocorticoid, bronchoscopy and thoracentesis were likewise higher in the mild-moderately elevated group than those in the normal group $(P<0.01)$. None of the patients needed mechanical ventilation. All the children recovered and were discharged from the hospital without death.

\section{Correlation Analysis of the Level of D-dimer With Different Variables}

Using the Spearman correlation test, we analyzed the relationships between the level of D-dimer and different variables. As shown in Figure 1, we found that the following variables showed significant positive correlations with the level of $\mathrm{D}$-dimer $(P<0.01)$ : N\%, CRP, $\mathrm{LDH}, \mathrm{CD}_{8}{ }^{+} \%$, IL-6, IL-10, IFN- $\gamma$, age, length of fever, length of stay, and length of antibiotic therapy. Meanwhile, L\%, PLT, PAB, $\mathrm{CD}_{4}{ }^{+} \%$, and TNF- $\alpha$ were negatively correlated with the level of Ddimer $(P<0.01)$. Among these variables, N\%, L\%, CRP, LDH, IL10 , length of fever, length of stay, and length of antibiotic therapy had particularly strong correlations with $\mathrm{D}$-dimer.

\section{DISCUSSION}

Mycoplasma pneumoniae (MP) is one of the most common pathogens of CAP in children. In some cases, MP infection will cause severe pneumonia with a variety of complications (Poddighe, 2018). Recently, a few researchers have also reported that thrombotic diseases could occur in patients with MPP, which is thought to be associated with the elevated level of Ddimer (Li et al., 2017; Liu et al., 2020; Mirijello et al., 2020). The elevation of D-dimer is widespread in patients with MPP, but the incidence of thrombosis is not high, and no case of embolism was found, as well as in our study. Furthermore, to the best of our knowledge, there is little published literature focusing on the significance of elevated D-dimer in patients with MPP. So, in the present study, we retrospectively enrolled $356 \mathrm{MPP}$ patients with different degrees of $\mathrm{D}$-dimer and analyzed the relationship between the levels of $\mathrm{D}$-dimer and clinical characteristics.

In our study, more severe signs and symptoms, higher incidence of extra-pulmonary complications, RMPP, SMPP, and longer process of disease were found in the elevated groups, especially in the severely elevated group. And there were more severe pulmonary lesions and higher levels of inflammatory biomarkers, such as CRP, $\mathrm{LDH}, \mathrm{N} \%, \mathrm{CD}_{8}{ }^{+} \%$, IL6 , IL-10, and IFN- $\gamma$, which were in line with the increase of Ddimer, whereas the $\mathrm{L} \%$ and $\mathrm{PAB}$ decreased with the levels of $\mathrm{D}$ dimer. Furthermore, we found that N\%, L\%, CRP, LDH, IL-10, length of fever, length of stay, and length of antibiotic therapy had strong correlations with D-dimer.

$\mathrm{D}$-dimer was originally known as a specific fibrin degradation product, as well as a specific marker of the fibrinolytic system, which can reflect the coagulation function and fiber activity of the body (Zhang et al., 2020). However, 


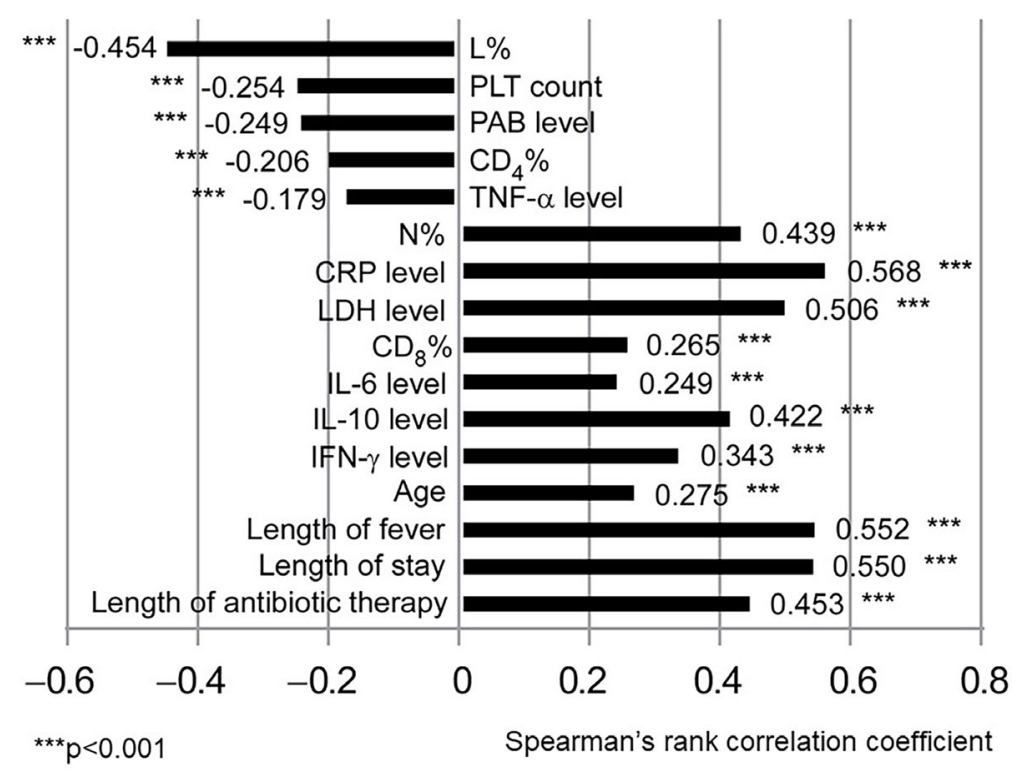

Figure 1. Correlation analysis of the level of D-dimer with different variables

FIGURE 1 | Correlation analysis of the level of D-dimer with different variables. Spearman rank-correlation coefficients were used to describe the association between different variables and D-dimer. Statistical significance was defined as $\mathrm{P}<0.05,{ }^{* \star *} \mathrm{P}<0.001$. N, neutrophil; L, lymphocyte; PLT, Platelet; CRP, C-reactive protein; LDH, Lactate dehydrogenase; PAB, Prealbumin; IL-6, Interleukin 6; IL-10, Interleukin 10; TNF- $\alpha$, Tumor necrosis factor-alpha; IFN- $\gamma$, Interferon-gamma.

recently, it has also been recognized as an indicator for evaluating the severity of CAP (Snijders et al., 2012). The results in our study showed that MPP patients with higher levels of D-dimer had more severe clinical manifestation and needed a longer duration of treatment, which might help to confirm other research's finding that D-dimer could act as an indicator for evaluating the severity of disease.

Cell-mediated immunological response plays a major role in the progression of MPP (Waites et al., 2017). Our preceding study and several other studies have demonstrated that inflammatory cytokines and some serum biomarkers were involved in the immunopathogenesis of MP infection (Tanaka et al., 2002; Narita and Tanaka, 2007; Lu et al., 2015; Choi et al., 2019; Ling et al., 2020). Furthermore, with the progress of MPP, immune cells release different kinds of inflammatory mediators, such as IL-1 $\beta$, IL-8, TNF- $\alpha$, and then aggravate the injury of vascular endothelial cells, leading to a significant increase of Ddimer (Iturriaga et al., 2015; Mishra et al., 2015; Jin et al., 2018). In this study, we found that some cytokines and inflammatory markers (CRP, LDH, N\%, $\mathrm{CD}_{8}{ }^{+} \%$, IL-6, IL-10, and IFN- $\gamma$ ) were significantly increased in the elevated groups, and there were highly positive correlations between the levels of D-dimer and some inflammatory markers (N\%, CRP, LDH, IL-10). These results implied that higher levels of $\mathrm{D}$-dimer might be associated with stronger inflammation, which was consistent with other reports (Yu et al., 2020).

MP is well recognized for producing a broad array of extrapulmonary manifestations. More interestingly, our study found that the incidence of extra-pulmonary complications was much higher in the elevated groups than that in the normal group, and the incidence of extra-pulmonary complications was increased with the level of $\mathrm{D}$-dimer. To our knowledge, this is the first report showing the relationship between the level of D-dimer and the extra-pulmonary complications in MPP patients. The mechanisms of MPP causing extra-pulmonary complications, by far, are not fully understood. Some studies have shown that it might be a direct effect of the MP that presents at the site of inflammation mediated by cytokine release by the host (Waites et al., 2017). Some researchers considered that it might be a direct or indirect effect by the production of vasculitis or thrombosis as a result of cytokines and chemokines or by immunomodulation through mediators, such as complement and fibrin D-dimers (Narita, 2009; Li et al., 2017; Waites et al., 2017). The latter might be the underlying mechanisms of the phenomenon in our study that the higher the incidence of extra-pulmonary complication was, the higher the $\mathrm{D}$-dimer value was. Recent medical literature also suggested that increased IgE levels (atopy) might be associated with extra-pulmonary manifestations in children with MPP (Wang et al., 2019). However, we found that there were no significant differences in IgE levels among all three groups in our study. This might be because that different age ranges of patients among groups caused different normal ranges of $\mathrm{IgE}$.

There were several limitations in our study. First, our study was a single-center retrospective study, which might have introduced a selection bias. The results reported in our study cannot be extrapolated to other areas of China. Thus, a prospective multicenter study is needed in the future. Second, 
there might be some patients who had coinfection with other pathogens, which could not be detected precisely and might therefore lead to the elevation of D-dimer. Third, we did not monitor dynamic changes of D-dimer. Therefore, it may result in some omissions of hypercoagulable states.

In conclusion, MPP patients with higher levels of D-dimer might have more severe clinical manifestation and need a longer duration of therapy, which was perhaps closely related to the degree of inflammation of MPP. These findings might help physicians to have deeper insights into MPP and provide proper treatment for MPP patients with higher levels of D-dimer.

\section{DATA AVAILABILITY STATEMENT}

The original contributions presented in the study are included in the article/supplementary material. Further inquiries can be directed to the corresponding authors.

\section{REFERENCES}

Adam, S. S., Key, N. S., and Greenberg, C. S. (2009). D-dimer antigen: current concepts and future prospects. Blood 113 (13), 2878-2887. doi: 10.1182/blood2008-06-165845

Chen, S., Ding, Y., Vinturache, A., Gu, H., Lu, M., and Ding, G. (2020). Pulmonary Embolism Associated With Mycoplasma in a Child. Lancet Infect. Dis. 20 (11), 1347. doi: 10.1016/S1473-3099(20)30253-X

Choi, Y. J., Jeon, J. H., and Oh, J. W. (2019). Critical Combination of Initial Markers for Predicting Refractory Mycoplasma Pneumoniae Pneumonia in Children: A Case Control Study. Respir. Res. 20 (1), 193. doi: 10.1186/s12931019-1152-5

Goldenberg, N. A., Knapp-Clevenger, R., and Manco-Johnson, M. J. (2004). Mountain States Regional Thrombophilia Group. Elevated plasma factor VIII and D-dimer levels as predictors of poor outcomes of thrombosis in children. N Engl. J. Med. 351 (11), 1081-1088. doi: 10.1056/NEJMoa040161

Huang, X., Li, D., Liu, F., Zhao, D., Zhu, Y., and Tang, H. (2021). Clinical Significance of D-Dimer Levels in Refractory Mycoplasma Pneumoniae Pneumonia. BMC Infect. Dis. 21 (1), 14. doi: 10.1186/s12879-020-05700-5

Iturriaga, R., Moya, E. A., and Del Rio, R. (2015). Inflammation and Oxidative Stress During Intermittent Hypoxia: The Impact on Chemoreception. Exp. Physiol. 100 (2), 149-155. doi: 10.1113/expphysiol.2014.079525

Jain, S., Williams, D. J., Arnold, S. R., Ampofo, K., Bramley, A. M., Reed, C., et al. (2015). Community-acquired pneumonia requiring hospitalization among U.S. children. N Engl. J. Med. 372 (9), 835-845. doi: 10.1056/NEJMoa1405870

Jin, X., Zhu, Y., Zhang, Y., Chen, J., Rong, L., and Zhao, X. (2018). Assessment of Levels of D-Dimer and Interferon- $\gamma$ in Pediatric Patients With Mycoplasma Pneumoniae Pneumonia and its Clinical Implication. Exp. Ther. Med. 16 (6), 5025-5030. doi: 10.3892/etm.2018.6873

Kang, B., Kim, D. H., Hong, Y. J., Son, B. K., Lim, M. K., Choe, Y. H., et al. (2016). Complete Occlusion of the Right Middle Cerebral Artery Associated With Mycoplasma Pneumoniae Pneumonia. Korean J. Pediatr. 59 (3), 149-152. doi: 10.3345/kjp.2016.59.3.149

Li, W., Fang, Y. H., Shen, H. Q., Yang, D. H., Shu, Q., and Shang, S. Q. (2017). Evaluation of a Real-Time Method of Simultaneous Amplification and Testing in Diagnosis of Mycoplasma Pneumoniae Infection in Children With Pneumonia. PloS One 12 (5), e0177842. doi: 10.1371/journal.pone.0177842

Ling, Y., Zhang, T., Guo, W., Zhu, Z., Tian, J., Cai, C., et al. (2020). Identify Clinical Factors Related to Mycoplasma Pneumoniae Pneumonia With Hypoxia in Children. BMC Infect. Dis. 20 (1), 534. doi: 10.1186/s12879-020-05270-6

Liu, J., He, R., Wu, R., Wang, B., Xu, H., Zhang, Y., et al. (2020). Mycoplasma Pneumoniae Pneumonia Associated Thrombosis at Beijing Children's Hospital. BMC Infect. Dis. 20 (1), 51. doi: 10.1186/s12879-020-4774-9

\section{AUTHOR CONTRIBUTIONS}

Conceived and designed the experiments: YYZ and ZMC. Wrote the manuscript: YZ and YYZ. Collected and analyzed the data: YZ, LLH, QNZ, MYL, YSW, YLZ and MXH. All authors contributed to the article and approved the submitted version.

\section{FUNDING}

This work was supported by grants from National Natural Science Foundation (81871264).

\section{ACKNOWLEDGMENTS}

We are grateful to colleagues in the Department of Pulmonology for taking care of patients and collecting the clinical data. We would like to thank the support of Zhejiang Provincial Key Laboratory of Immunity and Inflammatory diseases.

Li, T., Yu, H., Hou, W., Li, Z., Han, C., and Wang, L. (2017). Evaluation of Variation in Coagulation Among Children With Mycoplasma Pneumoniae Pneumonia: A Case-Control Study. J. Int. Med. Res. 45 (6), 2110-2118. doi: 10.1177/0300060517709613

Lu, A., Wang, C., Zhang, X., Wang, L., and Qian, L. (2015). Lactate Dehydrogenase as a Biomarker for Prediction of Refractory Mycoplasma Pneumoniae Pneumonia in Children. Respir. Care 60 (10), 1469-1475. doi: 10.4187/ respcare. 03920

Mélé, N., and Turc, G. (2018). Stroke Associated With Recent Mycoplasma Pneumoniae Infection: A Systematic Review of Clinical Features and Presumed Pathophysiological Mechanisms. Front. Neurol. 9, 1109. doi: 10.3389/fneur.2018.01109

Meyer Sauteur, P. M., Theiler, M., Buettcher, M., Seiler, M., Weibel, L., and Berger, C. (2020). Frequency and Clinical Presentation of Mucocutaneous Disease Due to Mycoplasma Pneumoniae Infection in Children With CommunityAcquired Pneumonia. JAMA Dermatol. 156 (2), 144-150. doi: 10.1001/ jamadermatol.2019.3602

Mirijello, A., La Marca, A., D’Errico, M. M., Curci, S., Vendemiale, G., Grandone, E., et al. (2020). Venous Thromboembolism During Mycoplasma Pneumoniae Infection: Case Report and Review of the Literature. Eur. Rev. Med. Pharmacol. Sci. 24 (19), 10061-10068. doi: 10.26355/eurrev_202010_23223

Mishra, K. P., Ganju, L., and Singh, S. B. (2015). Hypoxia Modulates Innate Immune Factors: A Review. Int. Immunopharmacol 28 (1), 425-428. doi: 10.1016/j.intimp.2015.07.008

Narita, M. (2009). Pathogenesis of Neurologic Manifestations of Mycoplasma Pneumoniae Infection. Pediatr. Neurol. 41 (3), 159-166. doi: 10.1016/ j.pediatrneurol.2009.04.012

Narita, M., and Tanaka, H. (2007). Cytokines Involved in the Severe Manifestations of Pulmonary Diseases Caused by Mycoplasma Pneumoniae. Pediatr. Pulmonol 42 (4), 397. doi: 10.1002/ppul.20445

Nowak-Göttl, U., and Kosch, A. (2004). D-Dimer, and Thromboembolism in Children. N Engl. J. Med. 351 (11), 1051-1053. doi: 10.1056/NEJMp048150

Park, S. J., Lee, Y. M., Lee, C. H., Cho, J. H., and Lee, J. H. (2012). A case of splenic infarction possibly attributable to Mycoplasma pneumoniae infection without accompanying pneumonia. J. Infect. Chemother. 18 (6), 945-947. doi: 10.1007/ s10156-012-0390-y

Poddighe, D. (2018). Extra-Pulmonary Diseases Related to Mycoplasma Pneumoniae in Children: Recent Insights Into the Pathogenesis. Curr. Opin. Rheumatol 30 (4), 380-387. doi: 10.1097/BOR.0000000000000494

Sakka, M., Connors, J. M., Hékimian, G., Martin-Toutain, I., Crichi, B., Colmegna, I., et al. (2020). Association Between D-Dimer Levels and Mortality in Patients With Coronavirus Disease 2019 (COVID-19): A Systematic Review and Pooled Analysis. J. Med. Vasc. 45 (5), 268-274. doi: 10.1016/j.jdmv.2020.05.003 
Snijders, D., Schoorl, M., Schoorl, M., Bartels, P. C., van der Werf, T. S., and Boersma, W. G. (2012). D-dimer levels in assessing severity and clinical outcome in patients with community-acquired pneumonia. A secondary analysis of a randomised clinical trial. Eur. J. Intern. Med. 23 (5), 436-441. doi: 10.1016/j.ejim.2011.10.019

Subspecialty Group of Respiratory Diseases (2013). The Society of Pediatrics, Chinese Medical Association; Editorial Board, Chinese Journal of Pediatrics. [Guidelines for Management of Community Acquired Pneumonia in Children (the Revised Edition of 2013) (I)]. Zhonghua Er Ke Za Zhi 51 (10), 745-752.

Tamura, A., Matsubara, K., Tanaka, T., Nigami, H., Yura, K., and Fukaya, T. (2008). Methylprednisolone Pulse Therapy for Refractory Mycoplasma Pneumoniae Pneumonia in Children. J. Infect. 57 (3), 223-228. doi: 10.1016/ j.jinf.2008.06.012

Tanaka, H., Narita, M., Teramoto, S., Saikai, T., Oashi, K., Igarashi, T., et al. (2002). Role of Interleukin-18 and T-Helper Type 1 Cytokines in the Development of Mycoplasma Pneumoniae Pneumonia in Adults. Chest 121 (5), 1493-1497. doi: 10.1378/chest.121.5.1493

Uehara, S., Sunakawa, K., Eguchi, H., Ouchi, K., Okada, K., Kurosaki, T., et al. (2011). Japanese Guidelines for the Management of Respiratory Infectious Diseases in Children 2007 With Focus on Pneumonia. Pediatr. Int. 53 (2), 264276. doi: 10.1111/j.1442-200X.2010.03316.x

Waites, K. B., and Talkington, D. F. (2004). Mycoplasma Pneumoniae and its Role as a Human Pathogen. Clin. Microbiol. Rev. 17 (4), 697-728. doi: 10.1128/ CMR.17.4.697-728.2004

Waites, K. B., Xiao, L., Liu, Y., Balish, M. F., and Atkinson, T. P. (2017). Mycoplasma Pneumoniae From the Respiratory Tract and Beyond. Clin. Microbiol. Rev. 30 (3), 747-809. doi: 10.1128/CMR.00114-16

Wang, Z., Sun, J., Liu, Y., and Wang, Y. (2019). Impact of Atopy on the Severity and Extrapulmonary Manifestations of Childhood Mycoplasma Pneumoniae Pneumonia. J. Clin. Lab. Anal. 33 (5), e22887. doi: 10.1002/jcla.22887

Weitz, J. I., Fredenburgh, J. C., and Eikelboom, J. W. (2017). A Test in Context: DDimer. J. Am. Coll. Cardiol. 70 (19), 2411-2420. doi: 10.1016/j.jacc.2017.09.024
Witmer, C. M., Steenhoff, A. P., Shah, S. S., and Raffini, L. J. (2007). Mycoplasma Pneumoniae, Splenic Infarct, and Transient Antiphospholipid Antibodies: A New Association? Pediatrics 119 (1), e292-e295. doi: 10.1542/peds.2006-1340

Yu, B., Li, X., Chen, J., Ouyang, M., Zhang, H., Zhao, X., et al. (2020). Evaluation of Variation in D-Dimer Levels Among COVID-19 and Bacterial Pneumonia: A Retrospective Analysis. J. Thromb. Thrombolysis 50 (3), 548-557. doi: 10.1007/ s11239-020-02171-y

Zhang, Y., Chen, Y., Chen, Z., Zhou, Y., Sheng, Y., Xu, D., et al. (2014). Effects of Bronchoalveolar Lavage on Refractory Mycoplasma Pneumoniae Pneumonia. Respir. Care 59 (9), 1433-1439. doi: 10.4187/respcare.03032

Zhang, Y., Mei, S., Zhou, Y., Huang, M., Dong, G., and Chen, Z. (2016). Cytokines as the Good Predictors of Refractory Mycoplasma Pneumoniae Pneumonia in School-Aged Children. Sci. Rep. 6, 37037. doi: 10.1038/srep37037

Zhang, L., Yan, X., Fan, Q., Liu, H., Liu, X., Liu, Z., et al. (2020). D-dimer levels on admission to predict in-hospital mortality in patients with Covid-19. J. Thromb. Haemost. 18 (6), 1324-1329. doi: 10.1111/jth.14859

Zhou, Y., Zhang, Y., Sheng, Y., Zhang, L., Shen, Z., and Chen, Z. (2014). More Complications Occur in Macrolide-Resistant Than in Macrolide-Sensitive Mycoplasma Pneumoniae Pneumonia. Antimicrob. Agents Chemother. 58 (2), 1034-1038. doi: 10.1128/AAC.01806-13

Conflict of Interest: The authors declare that the research was conducted in the absence of any commercial or financial relationships that could be construed as a potential conflict of interest.

Copyright (c) 2021 Zheng, Hua, Zhao, Li, Huang, Zhou, Wang, Chen and Zhang. This is an open-access article distributed under the terms of the Creative Commons Attribution License (CC BY). The use, distribution or reproduction in other forums is permitted, provided the original author(s) and the copyright owner(s) are credited and that the original publication in this journal is cited, in accordance with accepted academic practice. No use, distribution or reproduction is permitted which does not comply with these terms. 